\title{
Inhibition of proprotein convertase subtilisin/kexin type 9 attenuates neuronal apoptosis following focal cerebral ischemia via apolipoprotein $E$ receptor 2 downregulation in hyperlipidemic mice
}

\author{
LEI WANG ${ }^{1}$, ZI WANG ${ }^{1}$, JIANDANG SHI $^{2}$, QIAN JIANG ${ }^{1}$, HONG WANG $^{1}, \mathrm{XU} \mathrm{LI}^{1}$ and DI HAO ${ }^{1}$ \\ ${ }^{1}$ Cardiovascular and Cerebrovascular Drugs Research and Development Center, Tianjin Institute of Medical \\ and Pharmaceutical Sciences, Tianjin 300020; ${ }^{2}$ Department of Biochemistry and Molecular Biology, \\ College of Life Sciences, Nankai University, Tianjin 300071, P.R. China
}

Received January 3, 2018; Accepted July 12, 2018

DOI: $10.3892 /$ ijmm.2018.3797

\begin{abstract}
The inhibition of proprotein convertase subtilisin/kexin type 9 (PCSK9) protects a variety of cell types against neuronal apoptosis by binding to apolipoprotein $\mathrm{E}$ receptor 2 (ApoER2). The present study aimed to determine the association between PCSK9/ApoER2 signaling and neuronal apoptosis following middle cerebral artery occlusion (MCAO) injury in hyperlipidemic mice. For this purpose, C57BL/6 mice fed with a high-fat diet (HFD) for 6 weeks were exposed to MCAO. Subsequently, PCSK9 was inhibited by a lentiviral vector harboring short-hairpin RNA (shRNA) targeting PCSK9, which was stereotaxically injected into the cerebral cortex of mice. At $48 \mathrm{~h}$ post-ischemia, hematoxylin-eosin staining and a terminal deoxynucleotidyl transferase dUTP nick end labeling assay were performed to determine cerebral tissue injury and apoptosis. PCSK9 and ApoER2 expression levels were assessed by reverse transcription-quantitative polymerase chain reaction, immunohistochemistry and western blotting. The results indicated that hyperlipidemia and increased PCSK9 expression were evident in HFD mice. Cerebral histological injury and neuronal apoptosis, as well as PCSK9 and ApoER2 levels, which were increased upon ischemia in hyperlipidemic mice, were attenuated by PCSK9 shRNA treatment. These protective effects of PCSK9 shRNA interference were associated with decreased neuronal apoptosis and a reduced level of ApoER2 expression
\end{abstract}

Correspondence to: Professor Lei Wang, Cardiovascular and Cerebrovascular Drugs Research and Development Center, Tianjin Institute of Medical and Pharmaceutical Sciences, 79 Duolun Road, Heping, Tianjin 300020, P.R. China

E-mail: zws9905@sina.com

Key words: proprotein convertase subtilisin/kexin type 9, apolipoprotein E receptor 2, neuronal apoptosis, ischemic stroke, hyperlipidemia in the hippocampus and cortex. The data of the present study demonstrated that the PCSK9 shRNA-mediated anti-apoptotic effect induced by MCAO in hyperlipidemic mice is associated with ApoER2 downregulation, which may be a potential new therapy for stroke treatment in patients with hyperlipidemia.

\section{Introduction}

Ischemic stroke accounts for the majority of stroke cases, and is the leading cause of long-term disability and mortality worldwide (1). Emerging evidence has indicated that risk factors, including hypertension, diabetes and hyperlipidemia, are associated with an increased mortality rate in patients with ischemic stroke. Among the risk factors, hyperlipidemia is a major risk factor for ischemic stroke (2). The demonstrated efficacy of statin therapy in primary and secondary prevention of stroke by reducing serum low-density lipoprotein cholesterol (LDL-C) levels is well established $(3,4)$. However, previous studies have indicated that high hyperlipidemia is associated with a lower risk of post-stroke mortality (5-7). Despite these conflicting results, hyperlipidemia-associated ischemic stroke remains a major clinical challenge. The regulation of LDL receptor (LDLR) by proprotein convertase subtilisin/kexin type 9 (PCSK9) and the contribution of PCSK9 to cholesterol homeostasis are well accepted. Although PCSK9 was first identified as a plasma protein associated with hyperlipidemia just over a decade ago, a rich body of knowledge has been developed since, and therapeutic strategies inhibiting this target have been approved in numerous countries $(3,4,8)$. PCSK9 exhibits high affinity towards LDLR family members, including LDLR, very LDLR (VLDLR), apolipoprotein E receptor 2 (ApoER2), LDLR-related protein 1 (LRP1) and $\beta$-site amyloid precursor protein-cleaving enzyme 1 (BACE1) (9-11). These multiple ligand-receptor interactions have been reported to serve a well-established role in the systemic control of blood cholesterol, as well as a less characterized role in neuronal development and apoptosis (10). Previous studies revealed that PCSK9 is implicated in oxidized LDL-C-induced endothelial cell apoptosis, which is attenuated by PCSK9 silencing (12-14). 
PCSK9 serves a vital role in numerous processes resulting from hyperlipidemia, including free radical generation, lipid oxidation and inflammatory cell infiltration, which may be also involved in brain damage processes $(1,2)$. These pathological events ultimately lead to programmed cell death, also known as apoptosis, which is activated and maintained for several hours or days following stoke onset, thus contributing to cerebral ischemia injury $(1,15)$. Additionally, a series of previous cell studies confirmed the association between PCSK 9 and neuronal apoptosis (16-18). Thus, the present study hypothesized that PCSK9 may contribute to neuronal apoptosis in hyperlipidemia-associated ischemic stroke.

ApoER2 is expressed predominantly in the brain, and its role in neuronal survival depends on the pathological and physiological conditions. ApoER2 is required for protection against neuronal cell loss during normal ageing, while it selectively promotes neuronal cell death upon injury in the adult brain $(19,20)$. Although both PCSK9 and ApoER2 are implicated in brain injury, it remains controversial whether PCSK9 regulates the levels of ApoER2, and whether this has a functional significance in the brain (10). A number of previous in vitro studies have suggested that ApoER2 is the mediator of PCSK9-induced neuronal apoptosis (10), whereas other in vivo studies have proposed that PCSK9 does not regulate the levels of ApoER2 in the adult mouse brain $(10,11)$. Therefore, it is particularly important to determine the role of PCSK9 in hyperlipidemia-associated ischemic stroke and its impact on ApoER2 levels.

Considering the prevalence of stroke in hyperlipidemic patients, the present study aimed to clarify whether PCSK9 contributes to the exacerbation of ischemic brain apoptosis induced by middle cerebral artery occlusion (MCAO) injury in hyperlipidemic mice. Therefore, the present study investigated the influence of the inhibition of PCSK9 via injection of short hairpin RNA (shRNA) targeting PCSK9 on ischemic brain injury and apoptosis upon MCAO in hyperlipidemic mice. The study further explored the underlying mechanisms of action by focusing on the levels of ApoER2 in the hippocampus and cortex. The results suggested that PCSK9 contributed to hyperlipidemia/MCAO-induced brain injury by promoting neuronal apoptosis in the hippocampus and cortex, and the protective effect of PCSK9 shRNA was involved in the suppression of ApoER2.

\section{Materials and methods}

Ethics statement. The animal experiments were conducted in accordance with the National Institutes of Health Guide for the Care and Use of Laboratory Animals, and all the procedures were approved by the Animal Ethics Committee of Tianjin Institute of Medical and Pharmaceutical Sciences (Tianjin, China; approval no. IMPS-EAEP-Z-W2015KR04). All surgical procedures were performed under chloral hydrate anesthesia, and all efforts were made to minimize animal suffering.

High-fat diet (HFD). Male C57BL/6 mice (age, 9-10 weeks; weight, 24-26 g) were supplied by Beijing Vital River Laboratory Animal Technology Co., Ltd. (Beijing, China) and housed in a controlled environment $\left(25 \pm 1^{\circ} \mathrm{C}\right.$ and $40-70 \%$ humidity, with an artificial 12:12 h light/dark cycle). Mice were randomly assigned to the no-fat diet (NFD; n=8) or HFD $(n=40)$ groups. NFD mice were fed with a standard chow diet (cat. no. 11002900022675), while HFD mice were fed with an HFD consisting of $20 \%$ saccharose, $2 \%$ cholesterol, $15 \%$ lard and $0.3 \%$ cholate (cat. no. 11002900021707; Beijing Keao Xieli Feed Co., Ltd., Beijing, China). Food and water were available ad libitum for 6 weeks prior to surgery.

$M C A O$. Focal cerebral ischemia was induced by $\mathrm{MCAO}$ as previously described (21). Briefly, animals were deeply anesthetized with an intraperitoneal injection $10 \%$ chloral hydrate $(3.5 \mathrm{ml} / \mathrm{kg}$ body weight). Next, a silicone-coated nylon monofilament was inserted through a small incision in the right common carotid artery and was then advanced to $\sim 18 \mathrm{~mm}$ distal to the carotid bifurcation through the internal carotid artery in order to occlude the origin of the middle cerebral artery. In sham-operated animals, the same procedure was performed with the exception of inserting the intraluminal filament. To examine the critical role of PCSK9 in ischemic stroke, 32 HFD mice were randomly divided equally into four groups $(n=8)$, as follows: HFD-sham, HFD-MCAO, MCAO + LVRH1GP-null (shRNA-control), and MCAO + LVRH1GP-shRNA-PCSK9 (shRNA-PCSK9). Administration of the lentivirus expressing shRNA-PCSK9 or controls was performed immediately following the surgical procedure. Subsequent to continuous MCAO for $48 \mathrm{~h}$, the mice were sacrificed for further processing.

Lentivirus production and stereotaxic injection. Several recombinant lentiviral vectors harboring an shRNA sequence targeting PCSK9 (LVRH1GP-shRNA-PCSK9) were produced by GeneCopoeia, Inc. (Guangzhou, China). The lentiviruses harboring various shRNA sequences (Table I) against PCSK9 were injected into $\mathrm{C} 57 \mathrm{BL} / 6$ mice via the caudal vein in order to evaluate the interference efficiency in the kidney by reverse transcription-polymerase chain reaction (RT-PCR) (22). As shown in Table II, the results suggested that the PCSK9 shRNA sequence 3 provided the most reliable expression, and therefore this shRNA was selected for use in subsequent experiments. LVRH1GP-shRNA-PCSK9 or scramble shRNA (LVRH1GP-null) was delivered to the cortex of C57BL/6 mice via intracortical injection. The injection site was $1.2-\mathrm{mm}$ anterior to the bregma and $1.2-\mathrm{mm}$ lateral to the midline, and the injection depth was $3.0 \mathrm{~mm}$ (23). A total of $9 \mu \mathrm{l}$ purified lentivirus targeting PCSK9 or scramble shRNA was slowly injected at a speed of $0.5 \mu \mathrm{l} / \mathrm{min}$. The needle was carefully withdrawn $10 \mathrm{~min}$ after the injection to avoid reflux of the cerebrospinal fluid.

Serum parameters. Subsequent to feeding with HFD for 6 weeks, the concentration of triglycerides (TG), total cholesterol (TC), high-density lipoprotein cholesterol (HDL-C) and LDL-C in the serum were measured using the corresponding assay kits according to the manufacturer's protocol (cat. nos. A110-1, A111-1, A112-1 and A113-1, respectively; Nanjing Jiancheng Bio-Engineering Institute, Co., Ltd., Nanjing, China).

RT-quantitative PCR (RT-qPCR). The RT-qPCR was performed as previously reported (24). Briefly, total RNA 
Table I. Short hairpin RNA target sequences.

Sequence

name Symbol Location Length Target sequence

\begin{tabular}{llrll}
\hline Sequence 1 & Pcsk9 & 891 & 21 & ggaacctggagcgaattatcc \\
Sequence 2 & Pcsk9 & 964 & 21 & ggaggtgtatctcttagatac \\
Sequence 3 & Pcsk9 & 1,228 & 21 & ggagtttattcggaagagtca \\
Sequence 4 & Pcsk9 & 109 & 21 & ccgaaacctgatcctttagta \\
\hline
\end{tabular}

Table II. Screening the most effective sequence among four shRNA sequences against PCSK9 by quantitative reverse transcription-polymerase chain reaction.

Relative PCSK9

mRNA expression in kidney

\begin{tabular}{lcc}
\cline { 2 - 3 } Group & $24 \mathrm{~h}$ & $48 \mathrm{~h}$ \\
\hline NFD-C57BL/6 & 1 & 1 \\
HFD-C57BL/6 & 2.226 & 2.226 \\
HFD+PCSK9 shRNA control & 1.905 & 1.905 \\
HFD+PCSK9 shRNA sequence 1 & 1.717 & 1.625 \\
HFD+PCSK9 shRNA sequence 2 & 1.570 & 1.566 \\
HFD+PCSK9 shRNA sequence 3 & 1.357 & 1.304 \\
HFD+PCSK9 shRNA sequence 4 & 1.412 & 1.489
\end{tabular}

NFD, no-fat diet; HFD, high-fat diet; PCSK9, proprotein convertase subtilisin/kexin type 9; shRNA, short hairpin RNA.

was extracted from liver, hippocampus and cortex using TRIzol ${ }^{\circledR}$ reagent (Life Technologies; Thermo Fisher Scientific, Inc., Waltham, MA, USA) according to the manufacturer's protocol. The A260/A280 ratio was measured by a spectrophotometer to determine the purity and concentration of the RNA. Complementary DNA was synthesized from total RNA using Moloney Murine Leukemia Virus Reverse Transcriptase (Promega Corporation, Madison, WI, USA). Subsequently, qPCR was performed using the SYBR Premix Ex Taq ${ }^{\text {TM }}$ II kit (Takara Bio, Inc., Otsu, Japan). The reaction conditions were as follows: $95^{\circ} \mathrm{C}$ for $5 \mathrm{~min}, 95^{\circ} \mathrm{C}$ for $10 \mathrm{sec}, 60^{\circ} \mathrm{C}$ for $30 \mathrm{sec}$, $72^{\circ} \mathrm{C}$ for $32 \mathrm{sec}, 40$ cycles; the detection curve was carried out as follows: $95^{\circ} \mathrm{C}$ for $15 \mathrm{sec}, 60^{\circ} \mathrm{C}$ for $1 \mathrm{~min}, 95^{\circ} \mathrm{C}$ for $15 \mathrm{sec}$, $60^{\circ} \mathrm{C}$ for $15 \mathrm{sec}$. The specific primer pairs were as follows: PCSK9 forward, 5'-GCATCCACAACACCCCT-3' and reverse, 5'-CTGCCTCCGGACACTAA-3' (125 bp); $\beta$-actin, forward 5'-ACTCTGTGTGGATTGGTGGC-3', and reverse, 5'-AGA AAGGGTGTAAAACGCAGC-3' (155 bp). The messenger RNA (mRNA) expression levels of PCSK9 were normalized to those of the $\beta$-actin gene. Data analysis was performed using the $2^{-\Delta \Delta C t}$ method (25) for relative quantification.

Brain histopathology and immunohistochemistry (IHC). The mouse brain tissues were rapidly isolated and fixed in $4 \%$ paraformaldehyde. Next, the tissues were embedded in paraffin, and consecutive cross-sections of the brain were cut and stained with hematoxylin and eosin (H\&E). Histological evaluation was performed by an investigator who was blinded to the experimental groups. For IHC staining, tissue sections were dewaxed and rehydrated through a xylene and graded alcohol series, and the slides were then incubated for $10 \mathrm{~min}$ at $95^{\circ} \mathrm{C}$ in a citrate solution for antigen retrieval. Peroxidase (POD) clearing was performed prior to blocking for $2 \mathrm{~h}$ with $1 \%$ bovine serum albumin in PBS at room temperature. Subsequently, the tissue sections were incubated with polyclonal rabbit anti-PCSK9 and rabbit anti-ApoER2 antibodies (1:1,000; cat. nos. ab31762 and ab204112, respectively; Abcam, Cambridge, MA USA) at $4^{\circ} \mathrm{C}$ overnight, followed by incubation with a secondary antibody conjugated with horseradish POD (cat. no. SA1028; Boster Biological Technology, Ltd, Wuhan, China) at $37^{\circ} \mathrm{C}$ for $45 \mathrm{~min}$. Specific labeling was visualized with a 3,3'-diaminobenzidine (DAB) kit and appeared light yellow or tan-colored, while cell nuclei were counterstained with hematoxylin (blue staining). Images were captured with a microscope, and in each slide, eight random images of the mouse hippocampus and cortex were obtained to determine the intensity of staining. IHC staining, expressed as the relative positive expression, was quantified using gray values by HMIAS-2000 W image system (version 1.0; Wuhan Qianping Image Technology Co., Ltd., Wuhan, China). A high gray value indicated low content of protein, while low gray scale indicated high content.

Western blot analysis. Western blotting was used to measure the hippocampal and cortical levels of PCSK9 and ApoER2. Briefly, total protein was extracted using radioimmunoprecipitation assay buffer with a protease inhibitor (cat. nos. P0013 and ST506, respectively; Beyotime Institute of Biotechnology, Haimen, China). Protein levels were quantified using the bicinchoninic acid assay (cat. no. P0010, Beyotime Institute of Biotechnology, Haimen, China) according to the manufacturer's protocol. Protein samples were separated by $10 \%$ SDS-PAGE and then transferred to polyvinylidene fluoride membranes. The membranes were incubated with the aforementioned rabbit anti-PCSK 9 and rabbit anti-ApoER 2 primary antibodies $(1: 1,000)$ at $4^{\circ} \mathrm{C}$ overnight. Following washing in TBS with Tween-20, the membranes were incubated with a POD-conjugated anti-rabbit immunoglobulin G secondary antibody (1:5,000; cat. no. BA1003; Wuhan Boster Biological Technology, Ltd., Wuhan, China). Protein bands were visualized by enhanced chemiluminescence (GE Healthcare Life Sciences, Little Chalfont, UK). The protein expression levels were quantitatively analyzed with FluorChem version 2.0 software (ProteinSimple, San Jose, CA, USA). The protein expression levels of PCSK9 and ApoER2 were normalized to those of $\beta$-actin.

Terminal deoxynucleotidyl transferase dUTP nick end labeling (TUNEL) assay. A TUNEL assay was applied to identify the apoptotic cells with nuclear DNA fragmentation in the ischemic cerebral hemisphere according to the manufacturer's protocol (In Situ Cell Death Detection kit; cat. no. 11684817910; Roche Diagnostics, Basel, Switzerland) (22). Upon deparaffinization and rehydration, tissue sections were permeabilized with proteinase $\mathrm{K}(20 \mu \mathrm{g} / \mathrm{ml})$ at $37^{\circ} \mathrm{C}$ for $10 \mathrm{~min}$ 
and then incubated with TUNEL reaction mixture at $37^{\circ} \mathrm{C}$ for $2 \mathrm{~h}$ in a humidified chamber in the dark. Converter POD was next added and incubated at $37^{\circ} \mathrm{C}$ for $30 \mathrm{~min}$. Subsequent to rinsing with PBS (three times; 5 min each), the tissue sections were developed with DAB tetrahydrochloride and counterstained with Mayer's hematoxylin. In each section, eight random microscopic fields from the mouse hippocampus and cortex were analyzed (4 mice in each study group). The apoptosis ratio was presented as the percentage of TUNEL-positive cells (brown) in the total number of cell nuclei (blue). Bar plots for the mouse brain hippocampus and cortex regions were separately exhibited.

Statistical analysis. Data are expressed as the mean \pm standard deviation. Statistically significant differences were evaluated by unpaired Student's t-test for comparisons between two groups, or by one-way analysis of variance followed by a Duncan's multiple range test for multiple comparisons. $\mathrm{P}<0.05$ was considered to indicate a statistically significant difference. Statistical analyses were performed using SPSS version 18.0 software (SPSS Inc., Shanghai, China).

\section{Results}

PCSK9 is upregulated in the liver and brain of HFD-induced hyperlipidemic mice. After 6 weeks of HFD feeding, the serum TG, TC and LDL-C levels were significantly higher, whereas the serum HDL-C level was significantly lower, in HFD mice as compared with the levels in NFD mice (Fig. 1A). IHC staining revealed upregulation of PCSK9 in the liver and hippocampus of HFD mice after 6 weeks of HFD feeding (Fig. 1B and C). Furthermore, the mRNA levels of PCSK9, as determined by RT-qPCR, were significantly increased in the hippocampus and cortex of HFD mice (Fig. 1D). Taken together, these data indicated that there was significant PCSK9 overexpression in the liver and brain of hyperlipidemic mice.

PCSK9 is upregulated following MCAO in hyperlipidemic mice. Hyperlipidemic mice were subjected to MCAO surgery in order to imitate ischemic stroke, and the expression of PCSK9 in the cerebral hemisphere was detected after $48 \mathrm{~h}$ of MCAO by RT-qPCR, western blot and IHC analyses. As shown in Fig. 2, there was significant upregulation of PCSK9 mRNA and protein levels in the hippocampus and cortex of HFD-MCAO mice compared with those of HFD-sham mice. These results indicated that ischemia due to hyperlipidemia promoted PCSK9 upregulation.

Increased histological injury and apoptosis following MCAO in hyperlipidemic mice. H\&E staining of the brain tissues of mice subjected to $48 \mathrm{~h}$ of ischemia revealed nuclear shrinkage, neuronal vacuolization, neuronal loss and neutrophil infiltration (Fig. 3A). The quantification of apoptotic cells in the hippocampus and cortex revealed that MCAO resulted in a significant increase in apoptosis compared with that observed in the HFD-sham group (Fig. 3B-D).

PCSK9 shRNA ameliorates histopathological damage and apoptosis following MCAO in hyperlipidemic mice. As shown in Fig. 4, PCSK9 shRNA interference markedly decreased the PCSK9 protein levels in the hippocampus and cortex, as demonstrated by western blotting and IHC staining. Western blotting revealed that the protein level of PCSK9 was significantly lower in PCSK9 shRNA therapy group than those in the HFD-MCAO group and the analysis on the IHC staining indicated that the gray scale of PCSK9 in the PCSK9 shRNA pretreated group was higher than in the HFD-MCAO group. In order to further confirm the effects of PCSK9 shRNA in the hippocampus and cortex of hyperlipidemic-MCAO mice, histopathological damage and apoptosis were evaluated using H\&E and TUNEL staining, respectively. Consistently, PCSK9 shRNA treatment partially attenuated the ischemic damage by decreasing vacuolization and neutrophil infiltration compared with that observed in the HFD-MCAO group (Fig. 5A). Since PCSK9 potentiated neuronal apoptosis, suppression of PCSK9 significantly reduced neuronal death (10,16-18). As expected, in the present study, neuronal apoptosis resulting from ischemia stroke was significantly decreased in the hippocampus and cortex of PCSK9 shRNA-pretreated mice compared with that in HFD-MCAO mice (Fig. 5B-D). Overall, PCSK9 inhibition contributed to the reduced brain injury and anti-apoptotic effects observed following MCAO in hyperlipidemic mice.

PCSK9 shRNA inhibits the apoptosis in MCAO mice associated with ApoER2 downregulation. To investigate the role of the PCSK9/ApoER2 signaling pathway in MCAO-induced apoptosis, the expression levels of PCSK9 and ApoER2 in hippocampus and cortex were further evaluated. IHC staining demonstrated that pretreatment with PCSK9 shRNA simultaneously inhibited the expression of ApoER2 as presented in Fig. 6. Consistent with observations by IHC staining, western blot analysis for PCSK9 and ApoER2 in hippocampus and cortex revealed that the expression of ApoER 2 was significantly decreased in the PCSK9 shRNA treatment group compared with HFD-MCAO group (Fig. 6). Collectively, these data indicated that the anti-apoptotic effect triggered by PCSK9 shRNA interference was associated with ApoER2 downregulation.

\section{Discussion}

It has been well established that PCSK9 serves a key role in maintaining lipid homeostasis by affecting the systemic levels of LDLR and cholesterol uptake (18). Excessive PCSK9 expression in hepatocytes is linked to dyslipidemia, and inhibitors/silencers of PCSK9 are currently being tested in clinical trials for the treatment of hypercholesterolemia (18). Consistently, the present study clearly confirmed that HFD-induced hyperlipidemia coincided with increased PCSK9 expression in the liver. Notably, PCSK9 is also highly expressed in the central nervous system (CNS) and affects a wide range of neuronal functions (9-11). In the hippocampus and cortex, a marked elevation of PCSK9 mRNA levels was observed in the present study after 6 weeks of HFD feeding. Accordingly, hyperlipidemia led to PCSK9 overexpression in both the liver and brain.

Hyperlipidemia is known to be a risk factor for stroke (2). PCSK9, a proprotein convertase that binds to ApoER2, VLDLR, LDLR, BACE1 and LRP1, has been linked to hyperlipidemia and cell apoptosis (9-11). Previous studies have reported that PCSK9 is increased in the adult brain 

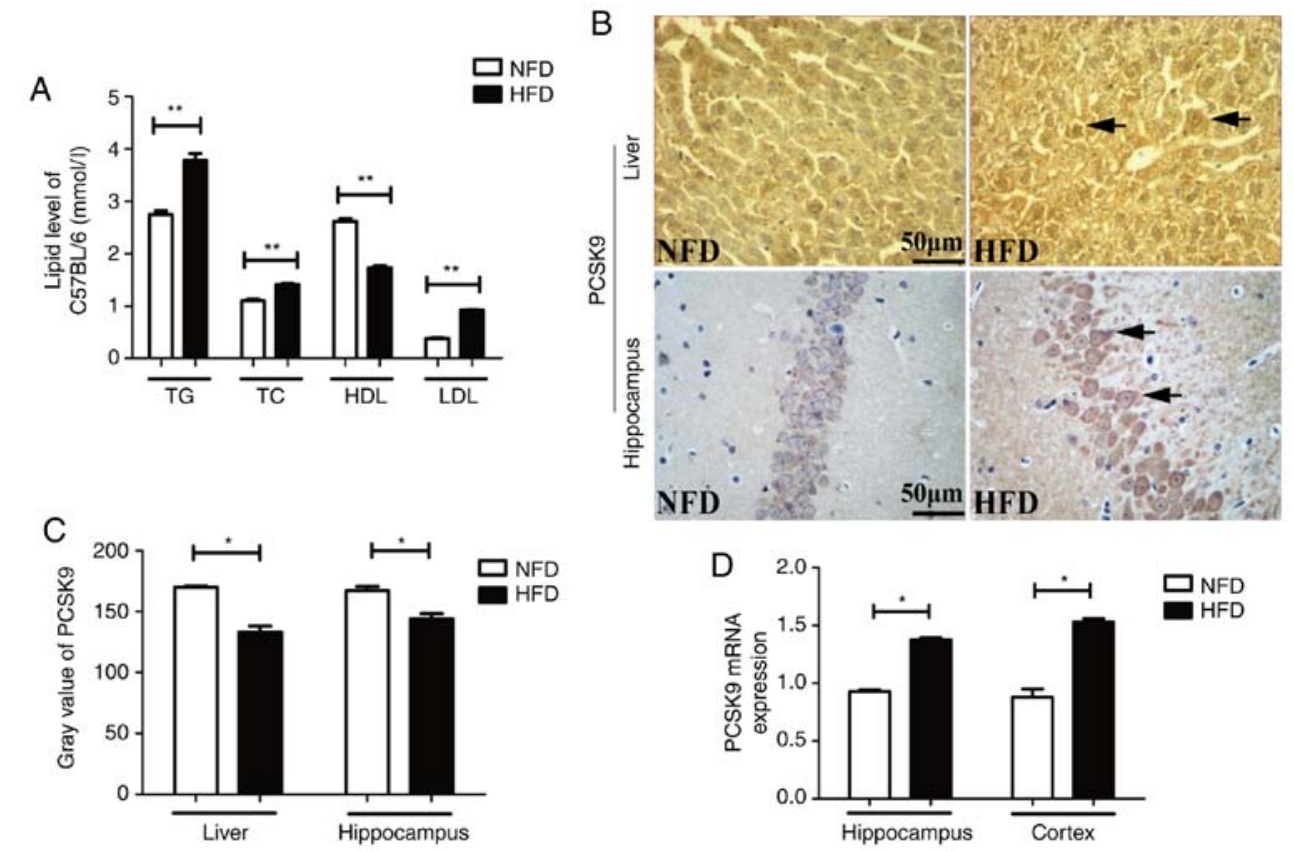

Figure 1. Blood lipids and PCSK9 expression levels were significantly increased upon HFD feeding. (A) Serum TG, TC and LDL-C levels were significantly increased, while HDL-C level was significantly decreased, in HFD mice compared with those in NFD mice ( $\mathrm{n}=8$ ). (B) Representative PCSK9 IHC staining in the liver and hippocampus tissues following HFD feeding. (C) Gray value of PCSK9 in IHC analysis was significantly decreased in the liver and hippocampus upon HFD feeding (n=4). (D) PCSK9 mRNA expression was significantly increased in the hippocampus and cortex following HFD feeding, according to reverse transcription-quantitative polymerase chain reaction analysis $(n=4)$. ${ }^{*} \mathrm{P}<0.05$ and ${ }^{* *} \mathrm{P}<0.01$ vs. NFD mice. PCSK9, proprotein convertase subtilisin/kexin type 9; HFD, high-fat diet; NFD, no-fat diet; TG, triglycerides; TC, total cholesterol; LDL-C, low-density lipoprotein cholesterol; HDL-C, high-density lipoprotein cholesterol; IHC, immunohistochemistry.

A

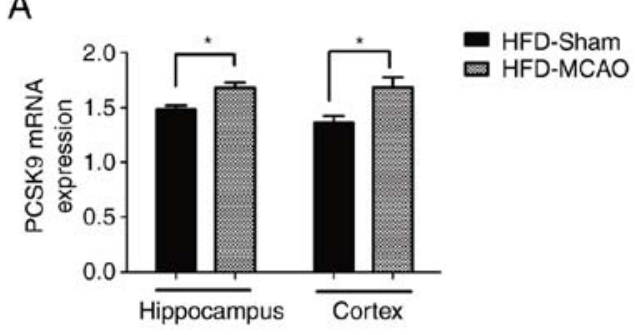

B

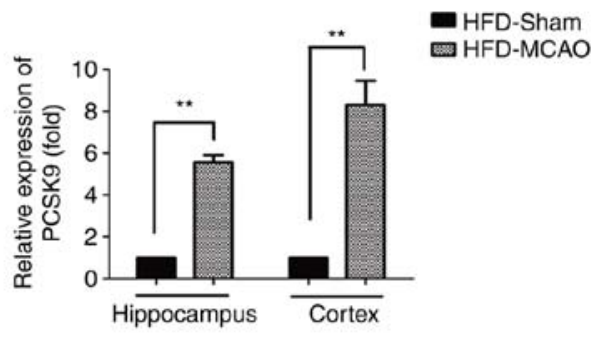

E

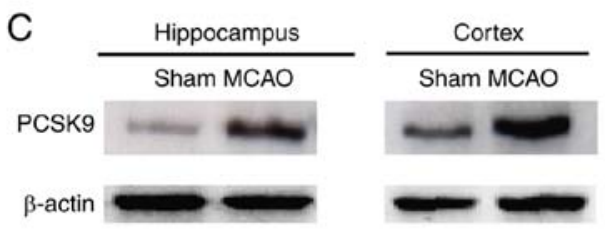

D
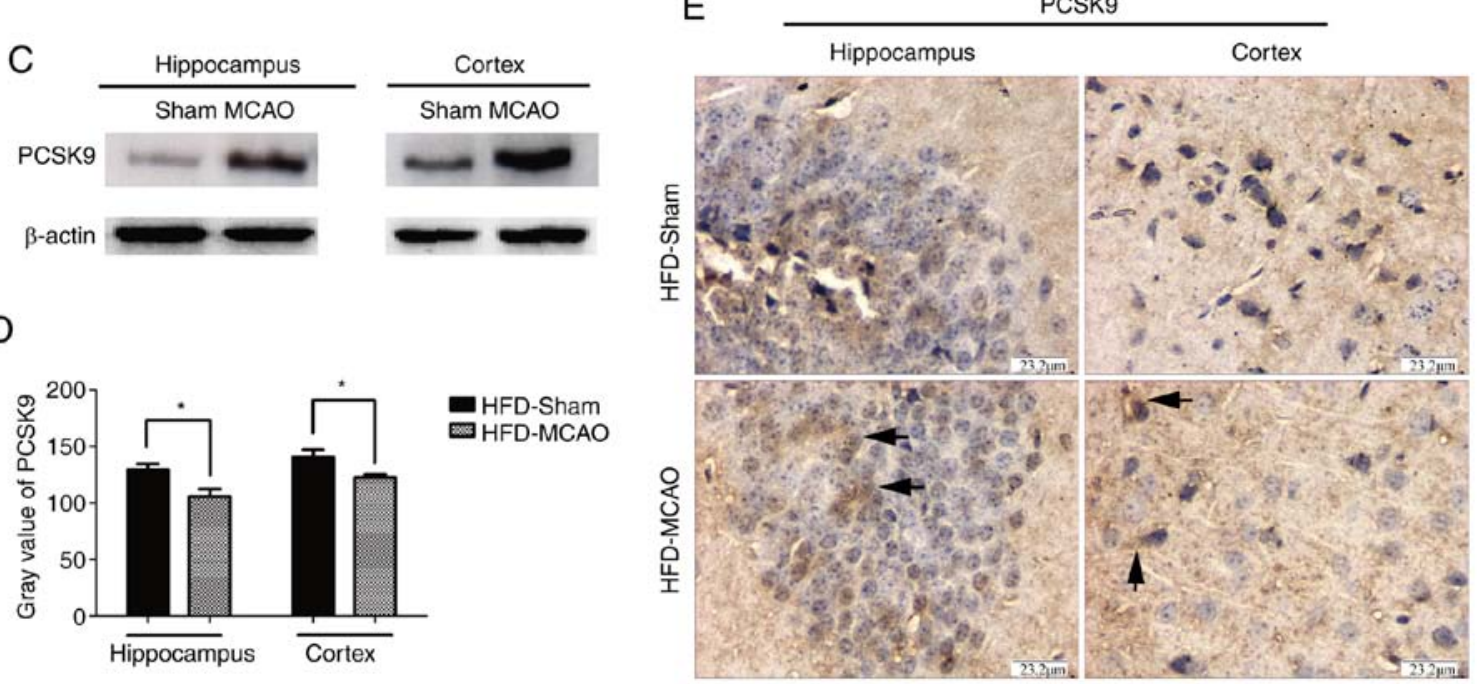

Figure 2. PCSK9 mRNA and protein levels were significantly increased following MCAO in hyperlipidemic mice. (A) mRNA and (B) protein levels of PCSK9 were significantly increased in HFD-fed mice subjected to MCAO, as demonstrated by reverse transcription-quantitative polymerase chain reaction and western blot analyses, respectively. (C) Representative western blotting image, displaying PCSK9 protein expression. (D) IHC analysis revealed that the gray value of PCSK9 was lower in the brain of HFD-MCAO mice compared with that in HFD-sham mice. (E) Representative images of IHC staining, showing PCSK9 protein expression. " $\mathrm{P}<0.05$ and ${ }^{* *} \mathrm{P}<0.01$ vs. HFD-sham mice $(\mathrm{n}=4)$. PCSK9, proprotein convertase subtilisin/kexin type 9; MCAO, middle cerebral artery occlusion; HFD, high-fat diet; IHC, immunohistochemistry. 

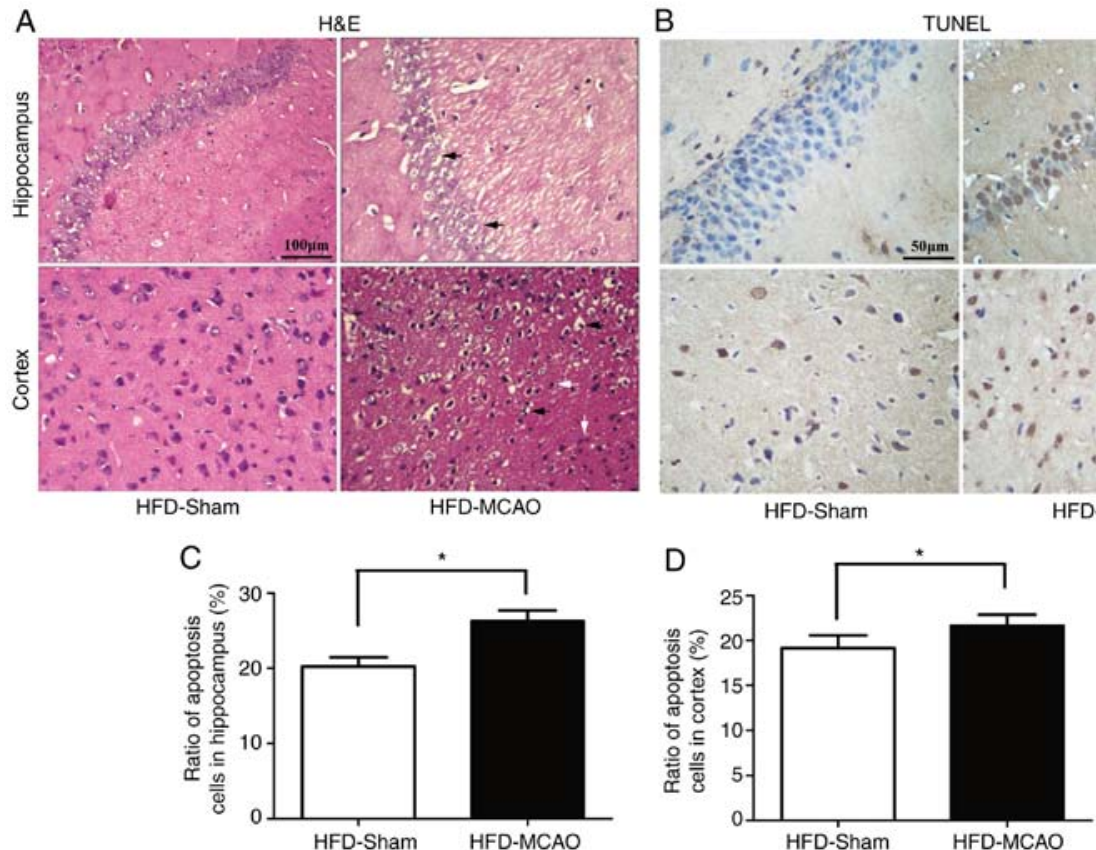

Figure 3. Ischemic injury and increasing apoptosis were observed in the hippocampus and cortex following middle cerebral artery occlusion in hyperlipidemic mice. (A) Representative images of the pyknotic cells and neuronal vacuolization in the hippocampus and cortex. (B) Representative images of the TUNEL-positive nuclei (brown) and total nuclei (blue). Bar plots demonstrating the percentage of TUNEL staining in the (C) hippocampus and (D) cortex. Black arrows indicate neuronal vacuolization and white arrows indicate inflammatory infiltration. ${ }^{*} \mathrm{P}<0.05$ and ${ }^{* *} \mathrm{P}<0.01$ vs. HDF-sham mice ( $\mathrm{n}=4$ ). TUNEL, terminal deoxynucleotidyl transferase dUTP nick end labeling; HFD, high-fat diet.
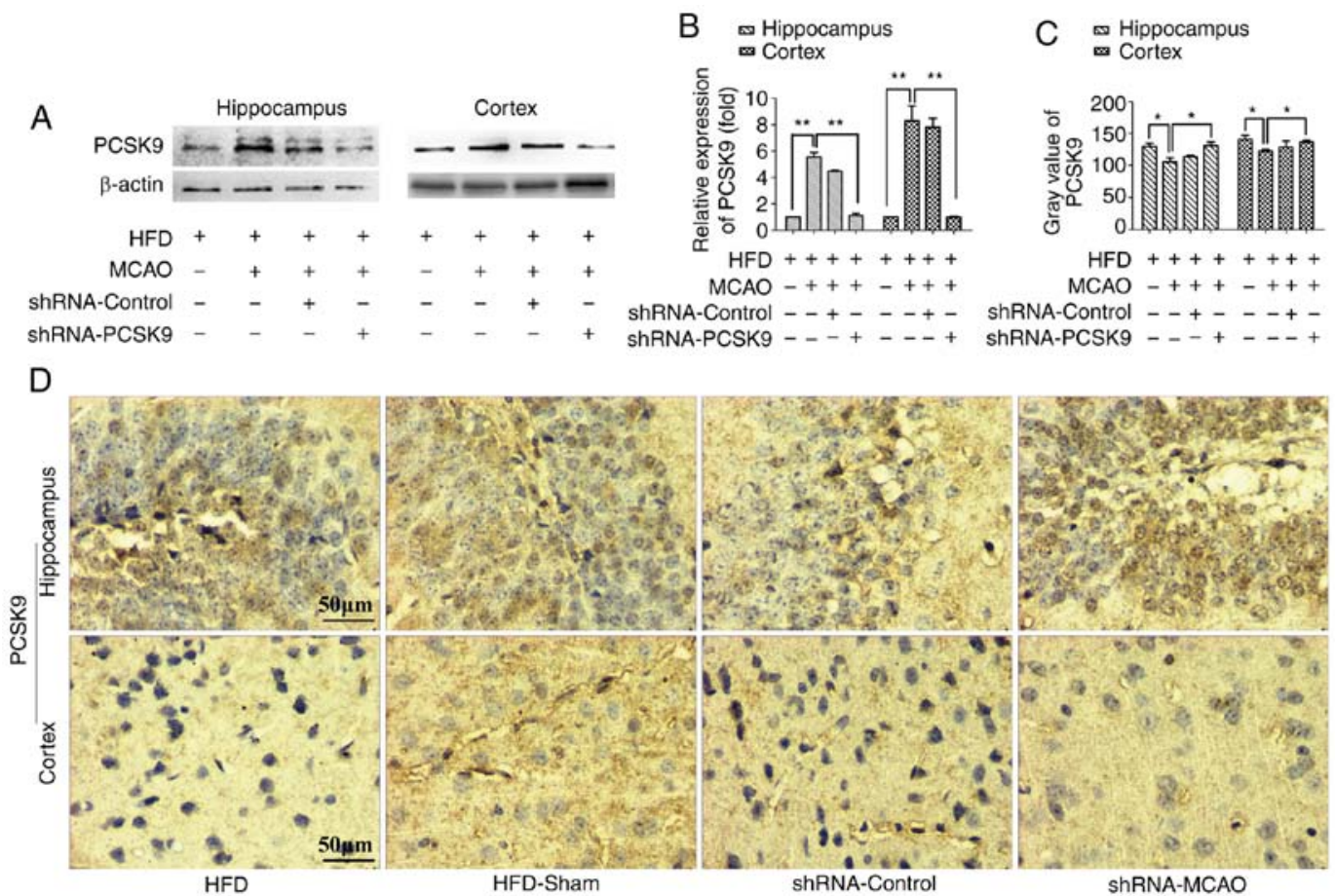

Figure 4. PCSK9 shRNA interference results in a reduction in the PCSK9 levels following MCAO in hyperlipidemic mice. (A) Representative western blot images demonstrating the protein levels of PCSK9 in the hippocampus and cortex. (B) The expression of PCSK9 in the hippocampus and cortex was significantly attenuated following PCSK9 shRNA treatment. (C) PCSK9 gray value in IHC staining of the hippocampus and cortex of animals exposed to PCSK9 shRNA was higher compared with that observed in the HFD-MCAO group. (D) Representative images of PCSK9 protein expression detected by IHC analysis." $\mathrm{P}<0.05$ and ${ }^{*} \mathrm{P}<0.01$ vs. HFD-MCAO mice $(\mathrm{n}=4)$. PCSK9, proprotein convertase subtilisin/kexin type 9; shRNA, short hairpin RNA; IHC, immunohistochemistry; HFD, high-fat diet; MCAO, middle cerebral artery occlusion.

following ischemia (18), while other in vitro studies revealed a key role for PCSK9 in neuronal apoptosis (10,16-18). In the present study, after 6 weeks of HFD feeding and subsequent establishment of an MCAO mouse model of ischemic stroke, it was demonstrated that PCSK9-dependent apoptosis served a major role in ischemic injury. Inhibition of PCSK9 reduced 


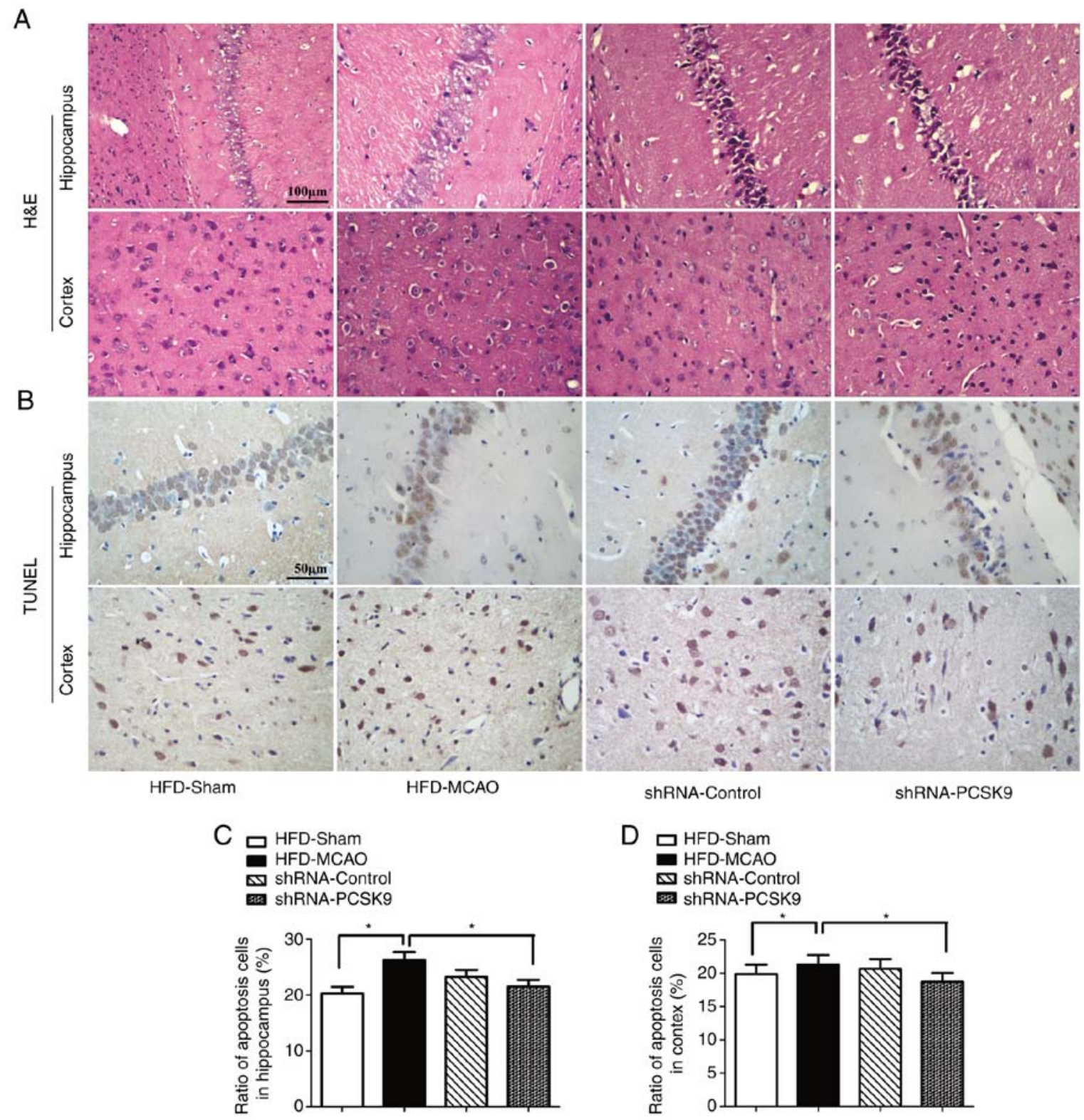

Figure 5. PCSK9 shRNA interference improves histological ischemic injury and apoptosis in the hippocampus and cortex following MCAO in hyperlipidemic mice. (A) Representative H\&E staining images, exhibiting histological injury and apoptosis in the hippocampus and cortex upon PCSK9 shRNA treatment. (B) TUNEL assay, indicating that the ratio of apoptotic cells in the (C) hippocampus and (D) cortex decreased following shRNA-PCSK9 treatment. ${ }^{*} \mathrm{P}<0.05$ and ${ }^{* *} \mathrm{P}<0.01$ vs. HFD-MCAO mice $(\mathrm{n}=4)$. PCSK9, proprotein convertase subtilisin/kexin type 9; shRNA, short hairpin RNA; HFD, high-fat diet; MCAO, middle cerebral artery occlusion; H\&E, hematoxylin and eosin; TUNEL, terminal deoxynucleotidyl transferase dUTP nick end labeling.

neuronal apoptosis in the hippocampus and cortex, which could also decrease brain damage. Thus, these findings suggest that PCSK9 regulated the apoptosis resulting from ischemic stroke in hyperlipidemic mice.

There are controversial reports regarding whether PCSK9 regulates the levels of ApoER2 in vivo possibly via neuronal apoptosis. A previous study reported that PCSK9 does not appear to modulate the expression of ApoER2 in the adult mouse brain $(10,11)$. By contrast, another study revealed that PCSK9 regulates neuronal apoptosis by adjusting ApoER2 signaling pathway in in vitro experiments using several types of neuronal cells (10). The present study demonstrated that suppression of PCSK9 attenuated neuronal death, which coincided with a reduction in the levels of ApoER2. This was in disagreement with previously published data demonstrating that PCSK9 inhibition resulted in elevated levels of ApoER2 (10). These conflicting results may be due to differences in the duration of feeding with a HFD, or time following MCAO when mice were examined, and experimental conditions, which are implicated in complex, multiple interactions among various pathological and physiological factors. Indeed, the role of ApoER2 in neuronal survival depends on pathophysiological changes. Notably, ApoER2 is required for protection against neuronal cell loss during normal ageing, but selectively promotes neuronal cell death upon injury $(19,20)$. In addition, ApoE is a major risk factor for several neurodegenerative diseases, and ApoER2 may also be relevant to the pathogenesis of Alzheimer's disease (19). The present results strongly support the hypothesis that the anti-apoptotic effect of PCSK9 inhibition is associated with downregulation of ApoER2. 

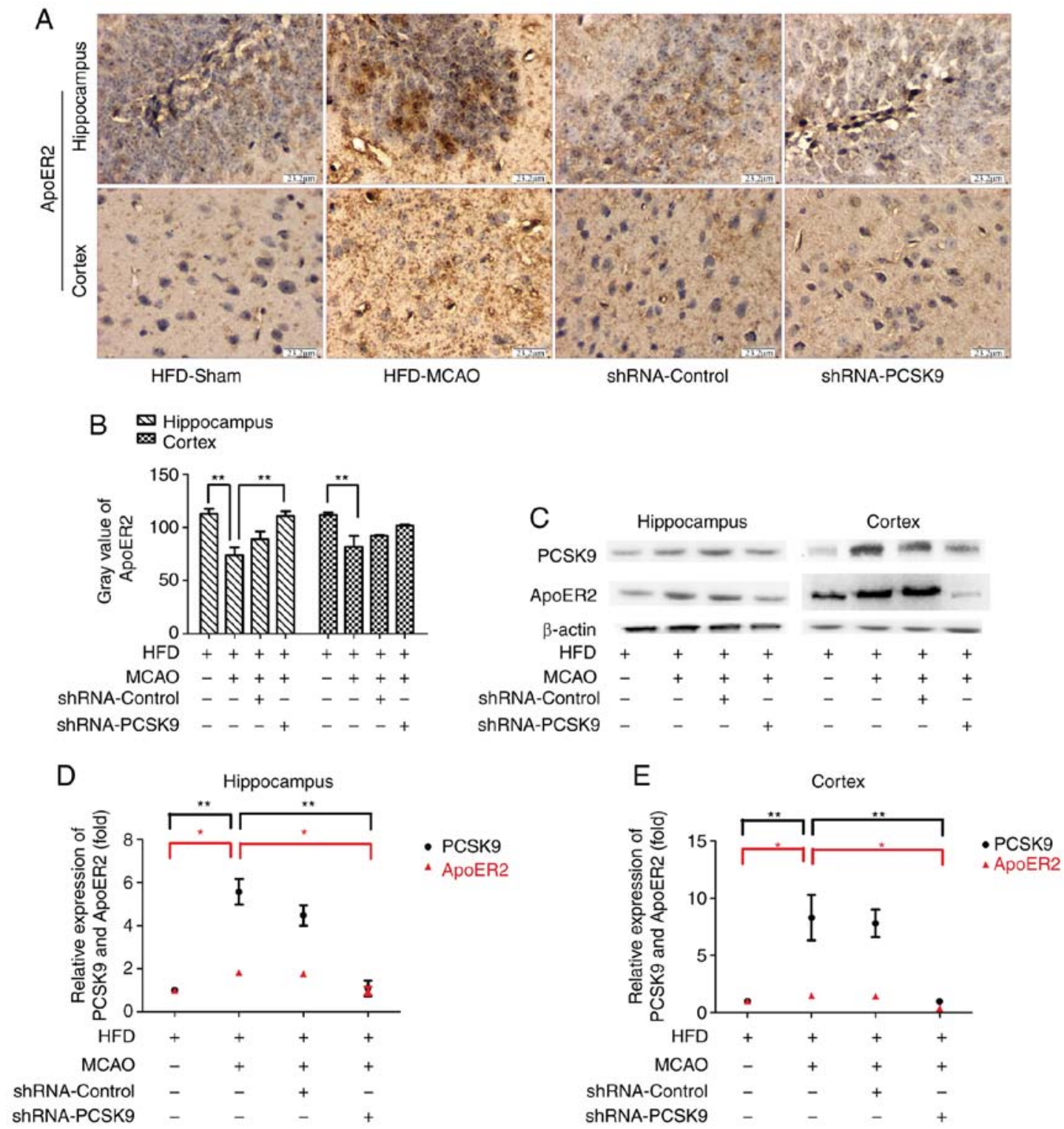

Figure 6. Anti-apoptotic effect of PCSK9, involving a reduction in ApoER2 levels subsequent to MCAO in hyperlipidemic mice. (A) Immunohistochemistry analysis for ApoER2 in the hippocampus and cortex. (B) Gray value of ApoER2 in the hippocampus and cortex was higher in PCSK9 shRNA mice compared with that in HFD-MCAO mice. (C) Representative western blot images of ApoER2 and PCSK9 protein expression levels in the hippocampus and cortex following PCSK9 shRNA treatment. Relative protein levels of PCSK9 and ApoER2 were concomitantly decreased in the (D) hippocampus and (E) cortex following shRNA-PCSK9 interference. ${ }^{*} \mathrm{P}<0.05$ and ${ }^{* *} \mathrm{P}<0.01$ vs. HFD-MCAO mice $(\mathrm{n}=4)$. PCSK9, proprotein convertase subtilisin/kexin type 9; ApoER2, apolipoprotein E receptor 2; shRNA, short hairpin RNA; HFD, high-fat diet; MCAO, middle cerebral artery occlusion.

However, the present study has several limitations. Firstly, although there is evidence for a role of hyperlipidemia in stroke, the results of several studies report that it is also associated with improved patient outcome following stroke as compared with that of individuals without hyperlipidemia (3-7). However, the current study has failed to demonstrate the comparison between HFD and NFD mice following MCAO with shRNA-PCSK9 interference. Furthermore, the findings of the present study indicate that the association of PCSK9 and ApoER2 are correlative and do not support a functional interaction between PCSK9 and ApoER2. However, this potential interaction remains to be determined by overexpression or silencing of ApoER2 in a further study. Finally, it is well known that PCSK9 binds to membrane-bound proteins, including LDLR, VLDLR, ApoER2 and BACE1, which are all highly expressed in the CNS (9-11). While downregulation of ApoER2 was observed upon treatment with shRNA against PCSK9 in the present study, downregulation of VLDLR and BACE1 may also occur. Thus, further research focusing on VLDLR and BACE1 would aid to determine whether partial loss of PCSK9 coincides with downregulation of ApoER2 specifically, or whether it occurs concurrently with VLDLR and BACE1 downregulation.

In conclusion, the findings of the current study implicate PCSK9 as a pro-apoptotic factor in ischemic brain injury in hyperlipidemic mice. The present study also demonstrated that the protective effect of shRNA against PCSK9 is involved in the suppression of ApoER2 expression. These data warrant further investigation of the therapeutic potential of PCSK9 inhibition treatment for ischemic stroke in hyperlipidemic patients. 


\section{Acknowledgements}

Not applicable.

\section{Funding}

The present study was supported by Tianjin Health Development Planning Commission of Science and Technology Fund Projects (grant no. 2015KR04) and the National Natural Science Foundation of China (grant no. 81202801).

\section{Availability of data and materials}

All date generated and/or analyzed during the current study are included in this published article.

\section{Authors' contributions}

LW and JS conceived and designed the study. ZW, QJ and HW performed the experiments. XL and DH analyzed the data and LW drafted the manuscript. All authors read and approved the final version of the manuscript.

\section{Ethics approval and consent to participate}

All experiments were approved by the Animal Care and Use Committee of Tianjin Institute of Medical and Pharmaceutical Sciences (Tianjin, China).

\section{Patient consent for publication}

Not applicable.

\section{Competing interests}

The authors declare that they have no competing interests.

\section{References}

1. Miao Y and Liao JK: Potential serum biomarkers in the pathophysiological processes of stroke. Expert Rev Neurother 14: 173-185, 2014.

2. Kim E, Tolhurst AT, Qin LY, Chen XY, Febbraio M and Cho S: CD36/fatty acid translocase, an inflammatory mediator, is involved in hyperlipidemia-induced exacerbation in ischemic brain injury. J Neurosci 28: 4661-4670, 2008.

3. Nozue T: Lipid lowering therapy and circulating PCSK9 concentration. J Atheroscler Thromb 24: 895-907, 2017.

4. Sun H, Krauss RM, Chang JT and Teng BB: PCSK9 deficiency reduces atherosclerosis, apolipoprotein B secretion and endothelial dysfunction. J Lipid Res 59: 207-223, 2018.

5. Yeramaneni S, Kleindorfer DO, Sucharew H, Alwell K, Moomaw CJ, Flaherty ML, Woo D, Adeoye O, Ferioli S, de Los Rios La Rosa F, et al: Hyperlipidemia is associated with lower risk of poststroke mortality independent of statin use: A population-based study. Int J Stroke 12: 152-160, 2017.

6. Zhao W, An Z, Hong Y, Zhou G, Guo J, Zhang Y, Yang Y, Ning $X$ and Wang J: Low total cholesterol level is the independent predictor of poor outcomes in patients with acute ischemic stroke: A hospital-based prospective study. BMC Neurol 16: 36, 2016.

7. Markaki I, Nilsson U, Kostulas K and Sjöstrand C: High cholesterol levels are associated with improved long-term survival after acute ischemic stroke. J Stroke Cerebrovasc Dis 23: e47-e53, 2014.
8. Shapiro MD and Fazio S: PCSK9 and atherosclerosis-lipids and beyond. J Atheroscler Thromb 24: 462-472, 2017.

9. Schulz R and Schlüter KD: PCSK9 targets important for lipid metabolism. Clin Res Cardiol Suppl 12 (Suppl 1): S2-S11, 2017.

10. Kysenius K, Muggalla P, Mätlik K, Arumäe U and Huttunen HJ: PCSK9 regulates neuronal apoptosis by adjusting ApoER2 levels and signaling. Cell Mol Life Sci 69: 1903-1916, 2012.

11. Liu M, Wu G, Baysarowich J, Kavana M, Addona GH, Bierilo KK, Mudgett JS, Pavlovic G, Sitlani A, Renger JJ, et al: PCSK9 is not involved in the degradation of LDL receptors and BACE1 in the adult mouse brain. J Lipid Res 51: 2611-2618, 2010.

12. Schulz R, Schlüter KD and Laufs U: Molecular and cellular function of the proprotein convertase subtilisin/kexin type 9 (PCSK9). Basic Res Cardiol 110: 4, 2015.

13. Li J, Liang X, Wang Y, Xu Z and Li G: Investigation of highly expressed PCSK9 in atherosclerotic plaques and ox-LDL-induced endothelial cell apoptosis. Mol Med Rep 16: 1817-1825, 2017.

14. Wu CY, Tang ZH, Jiang L, Li XF, Jiang ZS and Liu LS: PCSK9 siRNA inhibits HUVEC apoptosis induced by ox-LDL via Bcl/Bax-caspase9-caspase3 pathway. Mol Cell Biochem 359: 347-358, 2012.

15. Tang LL, Ye JY, Jiang SN and Zheng JS: 3,4-oxo-isopropylidene-shikimic acid inhibits cerebral ischemia-induced oxidative stress and neuronal apoptosis in rats. Am J Transl Res 9: 1764-1773, 2017.

16. Chiang LW, Grenier JM, Ettwiller L, Jenkins LP, Ficenec D, Martin J, Jin F, DiStefano PS and Wood A: An orchestrated gene expression component of neuronal programmed cell death revealed by cDNA array analysis. Proc Natl Acad Sci USA 98: 2814-2819, 2001.

17. Bingham B, Shen R, Kotnis S, Lo CF, Ozenberger BA, Ghosh N, Kennedy JD, Jacobsen JS, Grenier JM, DiStefano PS, et al: Proapoptotic effects of NARC 1 (=PCSK9), the gene encoding a novel serine proteinase. Cytometry A 69: 1123-1131, 2006.

18. Rousselet E, Marcinkiewicz J, Kriz J, Zhou A, Hatten ME, Prat A and Seidah NG: PCSK9 reduces the protein levels of the LDL receptor in mouse brain during development and after ischemic stroke. J Lipid Res 52: 1383-1391, 2011.

19. Beffert U, Nematollah Farsian F, Masiulis I, Hammer RE, Yoon SO, Giehl KM and Herz J: ApoE receptor 2 controls neuronal survival in the adult brain. Curr Biol 16: 2446-2452, 2006.

20. Waltmann MD, Basford JE, Konaniah ES, Weintraub NL and Hui DY: Apolipoprotein E receptor-2 deficiency enhances macrophage susceptibility to lipid accumulation and cell death to augment atherosclerotic plaque progression and necrosis. Biochim Biophys Acta 1842: 1395-1405, 2014.

21. Morris GP, Wright AL, Tan RP, Gladbach A, Ittner LM and Vissel B: A comparative study of variables influencing ischemic injury in the longa and koizumi methods of intraluminal filament middle cerebral artery occlusion in mice. PLoS One 11: e0148503, 2016.

22. Wang L, Wang Z, Yuan L, Hao D, Lü N and Li X: Shenfuqiangxin Capsule inhibits apoptosis through mitogen-activated protein kinase signal pathway in rats with cardio-renal syndrome induced by infrarenal aortic-clamping. J Tradit Chin Med 37: 80-87, 2017.

23. Shi W, Wei X, Wang Z, Han H, Fu Y, Liu J, Zhang Y, Guo J, Dong C, Zhou D, et al: HDAC9 exacerbates endothelial injury in cerebral ischaemia/reperfusion injury. J Cell Mol Med 20: 1139-1149, 2016.

24. Liu L, Wang Y and Yu Q: The PI3K/Akt signaling pathway exerts effects on the implantation of mouse embryos by regulating the expression of RhoA. Int J Mol Med 33: 1089-1096, 2014.

25. Livak KJ and Schmittgen TD: Analysis of relative gene expression data using real-time quantitative PCR and the 2(-Delta Delta C(T)) method. Methods 25: 402-408, 2001.

This work is licensed under a Creative Commons Attribution-NonCommercial-NoDerivatives 4.0 International (CC BY-NC-ND 4.0) License. 